\title{
Critical Trapping Conditions for Floating Liquid Marbles
}

\author{
Jing Jin, ${ }^{1}$ Kamalalayam Rajan Sreejith, ${ }^{1}$ Chin Hong Ooi, ${ }^{1}$ Dzung Viet Dao, ${ }^{1,2}$ and Nam-Trung Nguyen ${ }^{1, *}$ \\ ${ }^{1}$ Queensland Micro- and Nanotechnology Centre, Griffith University, Brisbane, Queensland 4111, Australia \\ ${ }^{2}$ School of Engineering and Built Environment, Griffith University, Gold Coast, Queensland 4222, Australia
}

(Dated: November 29, 2019)

\begin{abstract}
Floating liquid marbles, small nonwetting liquid droplets encapsulated by fine hydrophobic powder located on a free liquid surface, can serve as a unique micro bioreactor platform. Trapping and positioning these liquid marbles are essential for efficient sample handling. We investigate the dielectrophoretic trapping process of floating liquid marbles that are moving on a free water surface. The dynamic behavior of the trapping process is analyzed experimentally and theoretically. More importantly, a governing equation describing successful trapping is generalized from the energy balance. This study facilitates insights into dielectrophoretic manipulation of liquid marbles and fills the gap of knowledge in handling small soft systems.
\end{abstract}

\section{INTRODUCTION}

Microliter scale liquid droplets coated with multiple layers of hydrophobic micro- or nanoparticles are called liquid marbles (LMs). Compared to bare droplets, LMs possess various unique features [1-6]. They can stably rest or float on different solid and liquid substrates and even move across these surfaces with low loss, no leakage and relatively low evaporation [7-12]. These features make LMs the better alternative than droplets on a superhydrophobic surface for the storage and transport of a small amount of liquid [13, 14]. LMs floating on a carrier liquid can maintain their volumes for a long period of time. Furthermore, floating LMs can move with low friction and high accuracy, making them suitable for controlled manipulation, serving as a robust platform for digital microfluidics. Floating LMs have been successfully utilized to detect water pollution [15], gas emission [16, 17] and small trace of molecules [18]. Recently, LMs have been used as a novel bioreactor for culturing microorganisms or cells [19-22], as well as for amplification of deoxyribonucleic acid (DNA) [23, 24]. Key advantages of LMs as microreactors are the three-dimensional interior structure, good gas permittivity and low cross contamination. Automated manipulations of floating LMs, particularly precise trapping, are essential for implementing the tasks of sample preparation and detection in LM-based digital microfluidics. However, a comprehensive study on trapping of a moving and floating LM has not been conducted to date.

This letter reports experimentally and theoretically the trapping mechanism of a moving LM sliding on a free water surface under dielectrophoresis. We derive the critical condition for successful trapping of a moving LM. Compared to other actuation concepts for LMs [25-33], the dielectrophoretic (DEP) approach is a practical trapping concept for floating LMs due to the simplicity of the electrode setup and its good biocompatibility. DEP actuation allows for trapping, holding a LM at a specific location and releasing it to another location for further analysis. This technique could enable the further development of LM-based digital microfluidics or the concept of "lab in a marble".

* nam-trung.nguyen@griffith.edu.au

\section{MATERIALS AND METHODS}

Deionized water with a resistivity of $1.8 \times 10^{5} \Omega \mathrm{m}$ and polytetrafluoroethylene (PTFE) powder (Sigma-Aldrich, nominal diameter of $1 \mu \mathrm{m}$ ) serve as the liquid and the coating particles, respectively. The experimental setup consists of a floating LM with a volume of a few microliters and two cylindrical electrodes. The electrodes are connected to highvoltage DC-DC converters (Eastern Voltage Research HVPS 2 ) and suspended above the free water surface, Fig. 1. The sessile PTFE LM is first picked up by the lifting electrode at $4-\mathrm{kV}$ voltage and then released onto a smooth channel to roll toward the water surface $[34,35]$. The initial velocity of the LM on water is tuned by changing the rolling distance before reaching water. The injection angle of the $\mathrm{LM}$ ranges from $15^{\circ}$ to $60^{\circ} \pm 10^{\circ}$. LMs with volumes of $2.5,5,10,15$ and $25 \mu \mathrm{L}$ are selected for the trapping experiments. The working distance and the applied voltage of the trapping electrode are fixed at $30 \mathrm{~mm}$ and $5 \mathrm{kV}$, respectively. The trapping processes are recorded from the bottom of the setup using a high-speed camera (Photron Fastcam SA3) at 125 frames/second and an image size of $768 \times 768$ pixels.

After touching and sliding on the water surface, the LM experiences an attractive DEP force induced by the trapping electrode. This restoring force guides the LM toward the center line of the electrode. The LM travels via the increasing gradient of the nonuniform electric field, resulting in a spiral movement. The trapping process of the floating LM can be regarded as a two-dimensional (2D) problem, neglecting the small change of the effective marble height over the water surface. We then formulate an analytical model based on the modified point-charge assumption and the method of images to describe the nonuniform electric field. The simulated trajectory of the floating LM is then compared with experimental data. A MATLAB (MathsWorks) code evaluates the recorded video and derives the experimental trajectory. The overarching aim of this study is obtaining a universal model for DEP trapping of floating LMs with a set of initial conditions. This model will provide a guidance for controlled manipulation of LM-based reactors for chemical and biological assays. 

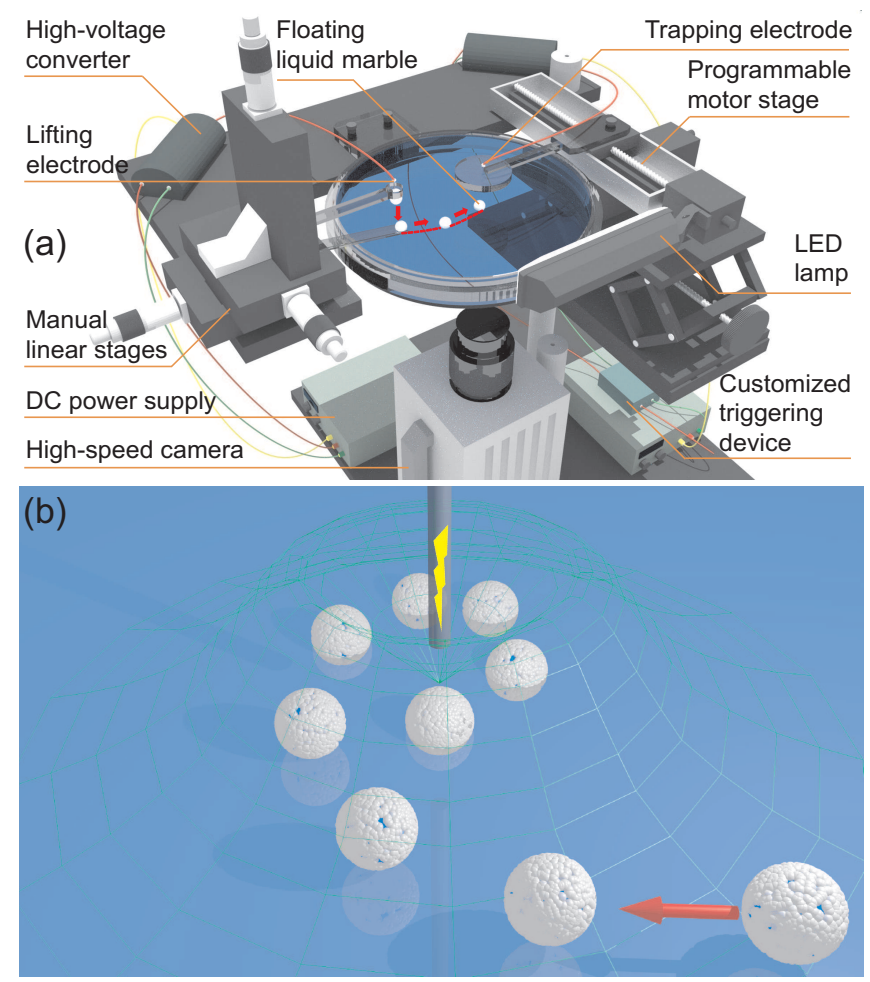

FIG. 1. Schematic diagrams of DEP trapping of a floating LM that moves on a free liquid surface. (a) The experimental setup. (b) The trapping concept.

\section{THEORY}

We first utilize a modified point-charge model to describe the electric field in the vicinity of the trapping electrode [3638]. For a hypothetical point charge suspended above the water/air interface, the electric potential experienced by a floating $\mathrm{LM}$ at the position $(r, z)$ can be described as:

$$
\begin{aligned}
& \Phi(q, d, r, z)=\Phi(V, d, r, z) \\
& \quad \cong V h\left[\frac{1}{\left[r^{2}+(d-z)^{2}\right]^{1 / 2}}-\frac{1}{\left[r^{2}+(d+z)^{2}\right]^{1 / 2}}\right] \\
& \quad=V h \sigma
\end{aligned}
$$

where $q$ is the electric charge, $V$ is the trapping voltage, $d$ is the virtual height of the point charge above the grounded water surface and has a linear relationship with the physical distance $h$ from the trapping electrode to the water surface ( $h \approx 8 \mathrm{~mm}$ ), and $\sigma$ represents the spatial component of the electric potential field. A correction factor will be added as this approach is only an approximation of the actual electric potential.

In each experiment with a specific marble volume, the applied voltage of $5 \mathrm{kV}$ and other parameters $V, h, d$ and $z$ remain constant. The potential distribution just above the water surface is $2 \mathrm{D}$ and only changes with $r(x, y)$. Consequently, the electric field strength $E$ along the horizontal direction can be calculated and simplified as:

$$
\begin{aligned}
\nabla E_{r}^{2} & =\nabla(-\nabla \Phi)^{2}{ }_{r}=(V h)^{2} \nabla(-\nabla \sigma)^{2}{ }_{r} \\
\cong & \frac{(V h)^{2} C d^{2} r}{\left(r^{2}+d^{2}\right)^{4}}=C(V h d)^{2} \psi(r),
\end{aligned}
$$

where $C$ is the correction factor to the analytical model that can be determined experimentally, and $\psi(r)$ represents the position component containing horizontal distance $r$.

The horizontal component of the DEP force for trapping a floating LM can be analytically described as:

$$
\begin{aligned}
F_{\mathrm{DEP}}(r) & =2 \pi \varepsilon_{m} R^{3} \nabla E^{2}{ }_{r}=2 \pi C \varepsilon_{m} R^{3}(V h d)^{2} \psi(r) \\
& =\frac{3 C \varepsilon_{m}}{2 \rho} m(V h d)^{2} \psi(r) .
\end{aligned}
$$

where $\varepsilon_{m}$ is the permittivity of air with a value of approximately $8.85 \times 10^{-12} \mathrm{~F} / \mathrm{m}$, and $R, \rho$ and $m$ are the radius, density $\left(\approx 1000 \mathrm{~kg} / \mathrm{m}^{3}\right)$ and mass of the undeformed floating LM, respectively.

To determine the DEP potential energy $U_{\text {DEP }}$ of the floating $\mathrm{LM}$ at a specific position, we set the infinite position as the reference point of zero potential energy and carry out the definite integration with the simplified DEP force expression to match the conversion between the work done by DEP restoring force and DEP potential energy, namely $U_{\mathrm{DEP}}(r)=$ $-W_{r e f \rightarrow r}=-\int_{\infty}^{r} F_{\mathrm{DEP}}(r) d r$. Combining Eq. (3) for the attractive DEP force that does positive work in the sliding motion of the floating LM, the corresponding DEP potential energy should always be negative, as depecited by the red solid line in Fig. 2. Thus, the relative total energy of floating LMs at different instances could be positive, zero or even negative.

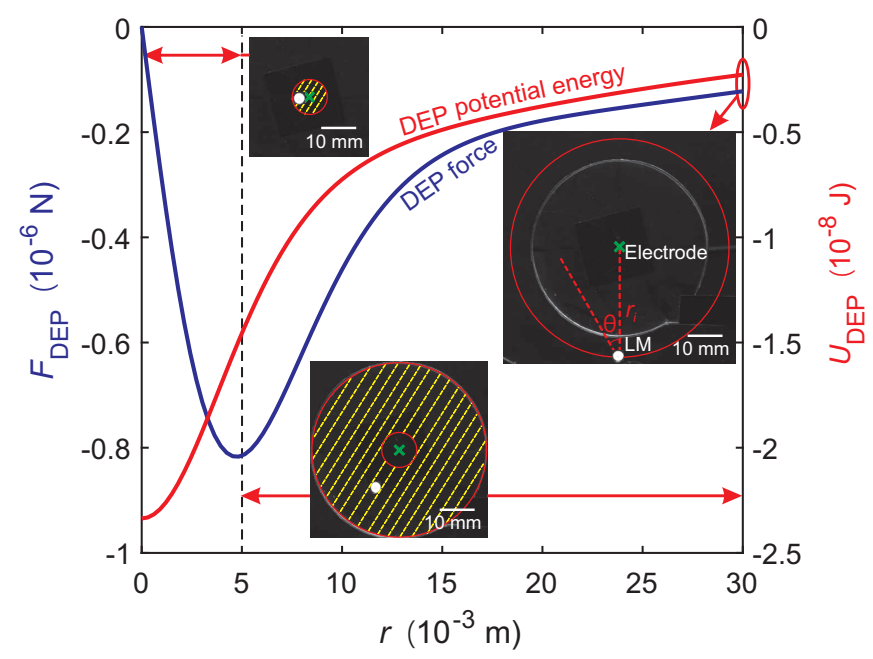

FIG. 2. The horizontal DEP force exerted on a $10-\mu \mathrm{L}$ floating $\mathrm{LM}$ and its DEP potential energy at various positions. 


\section{RESULTS AND DISCUSSION}

\section{A. Analysis on marble motion regimes}

After establishing the DEP potential energy, we use the energy balance analysis to describe the 2D DEP trapping process. Analyzing the force balance is not easy for various positions of the trajectory because of the changing force directions. The position where the sessile LM is injected onto the water surface is selected as the initial position $r_{i}$. By systematically comparing the marble motion paths in all experimental cases, we deduce that there should be at least one critical position that affects marble trapping at a specific set of initial conditions. If moving with a large initial velocity and any injection angles, the floating LM would slide quickly to a position where the velocity direction is approximately perpendicular to the direction of the attractive DEP force. This position is considered as the critical point $r_{c}$, which has the shortest distance to the electrode center line since the start of the injection process, Figs. 3(a) and 3(b).
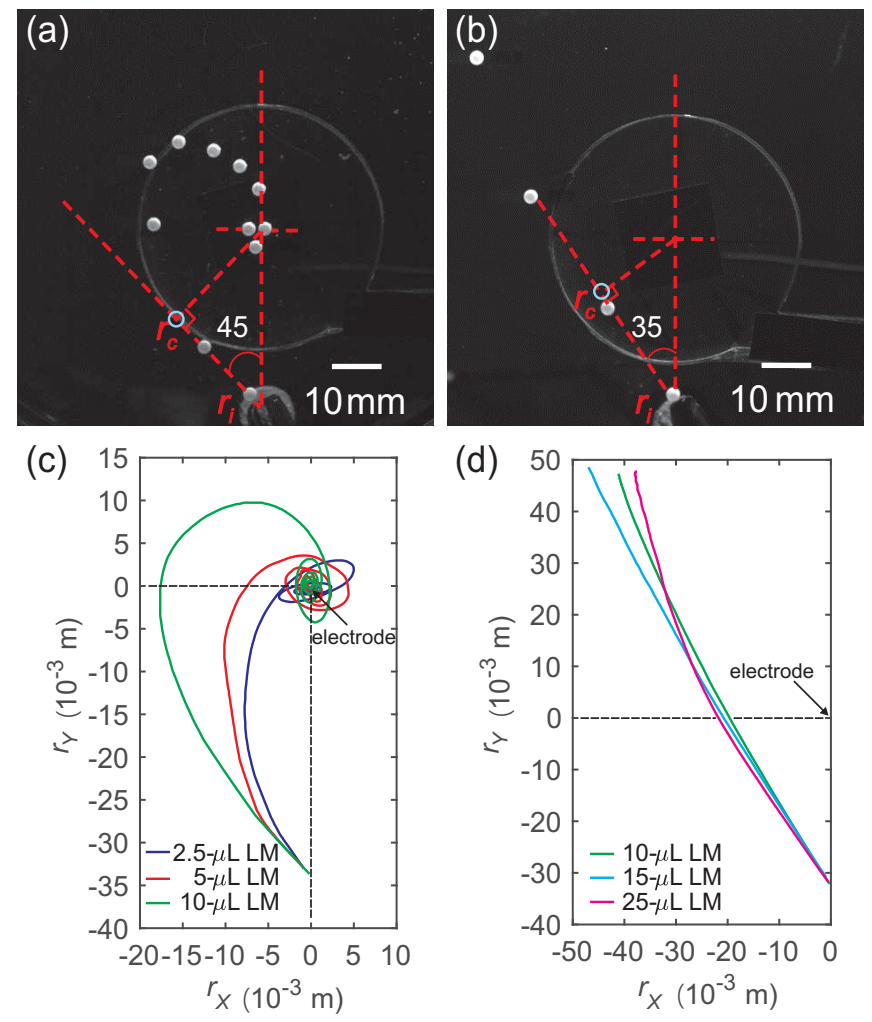

FIG. 3. Image sequences of $10-\mu \mathrm{L}$ floating LMs trapped along a spiral path (a) and escaping quickly (b) under DEP force in two typical experimental cases. (c),(d) Different trajectories of nonstationary floating LMs on a free water surface in trapping and escaping regimes (the electrode centroid position is set as the original point).

In general, there are two types of motion regimes induced by the nonuniform electric field around an electrode with 5$\mathrm{kV}$ applied voltage: trapping and escaping. Appendix A provides representative videos of these processes. The moving LMs with an initial velocity less than $0.1 \mathrm{~m} / \mathrm{s}$ and an injection angle of less than $38^{\circ}$ could easily reach the trapping electrode through a spiral movement. Increasing the size of the marble velocity or the injection angle allows floating LMs to escape. Once the LM escapes, it decelerates until a complete stop due to friction. It is noteworthy that a floating LM that escapes but then stops within the effective working range of the DEP force (normally $40 \mathrm{~mm}$ at $5-\mathrm{kV}$ trapping voltage) could subsequently be attracted back under the electrode. In a successful trapping case, the whole process usually completes within a few seconds. The floating LMs slide smoothly with few rotations in trapping and do not cause much interference to the carrier water surface. The LM escapes only, if both the initial velocity and the injection angle exceed their respective threshold values. In the experimental case of escaping and subsequent trapping, the trapping process is similar to the simple one-dimensional case from a stationary initial position [36]. The LM accelerates toward the center line of the trapping electrode and then oscillates around it until a complete stop.

Interestingly, almost all successful trapping cases with various parameters show a similar trajectory, which has a large curvature radius in the outer working ranges (over $10 \mathrm{~mm}$ to the electrode center line), Fig. 3(c). These trapping cases then show high-density path circles within the "real" effective working distance of $5 \mathrm{~mm}$, especially for the large floating LMs with a Bond number Bo of over 0.25. This finding supports the description of the inhomogeneous electric field. The Bond number of a LM is defined as Bo $=\rho g R^{2} / \gamma_{\mathrm{eff}}$, where $\gamma_{\text {eff }}$ is the effective surface tenison of the LM. However, the accurate measurement of the effective surface tension of LMs remains a challenge due to technical limitations [39-42]. In this study, a LM that floats on a carrier liquid deforms, and the coating layer is sandwiched between the liquid core and the liquid carrier. This arrangement affects the distribution of coating particles on the surface of the LM, and consequently influences its effective surface tension. Futhermore, the rapid motion of the floating LM under dielectrophoresis makes the related measurement extremely difficult. Here, we can only estimate the effective surface tension of floating LMs using the puddle height method introduced by Aussillous and Quéré in 2006 [2] $\gamma_{\mathrm{eff}}=h_{\mathrm{p}}{ }^{2} \rho g / 4$, where $h_{\mathrm{p}}$ is the height of a large LM with a puddle shape resting on a solid substrate. From our previous work [43], the effective surface tension of the LM is determined to be $0.068 \pm 0.004 \mathrm{~N} / \mathrm{m}$ for LMs with volumes ranging from 2.5 to $25 \mu \mathrm{L}$, similar to the measurement done by Arbatan and Shen [44], where the same PTFE powder is used. With the effective surface tension, the corresponding Bond number can be roughly calculated for the dimensionless analysis. In a typical escaping case, the floating LM moves quickly away from the electrode, which follows a straight trajectory. Only large LMs indicate a small curvature at the critical point (Fig. 3(d)), validating that the DEP force is proportional to the volume of the LM.

As for the floating LM travels head on toward the electrode centroid along the direction of the DEP force, it is different from the oblique trapping with a certain injection angle. The LM directly accelerates toward and then overshoots the position right below the electrode centroid with a maximum ve- 
locity. The maximum velocity in turn decides whether the LM can move out of the effective working range of DEP force. Enough initial kinetic energy may enable escaping. However, in all cases under investigation, there is no strictly head-on trapping case as the floating LM cannot strictly move along a straight path. Thus, the head-on trapping of a floating LM is not discussed in this paper.

\section{B. Energy-based dynamic governing equation for DEP trapping of floating liquid marbles}

We apply the conservation of energy to consider the initial point $r_{i}$ and the critical point $r_{c}$ in the movement of a floating LM with the kinetic energy, DEP potential energy and friction energy loss:

$$
\frac{1}{2} m v_{i}^{2}+U_{\mathrm{DEP}, i}=\frac{1}{2} m v_{c}^{2}+U_{\mathrm{DEP}, c}+U_{\mathrm{loss}},
$$

The relation between the kinetic energy and the DEP potential energy of the floating LM at this critical point directly decides whether the marble can be trapped or not. At the boundary between trapping and escaping, the escaping velocity $v_{e}$ in the nonuniform electric field should meet the condition that the total energy of the floating LM infinitely approaches zero: $\frac{1}{2} m v_{e}^{2}+U_{\mathrm{DEP}, c}=0$. The value of $v_{e}$ is found to be independent of the marble mass. If the injection angle is set, an escaping velocity ranging from 0.022 to $0.063 \mathrm{~m} / \mathrm{s}$ will be decided for all marble volumes investigated in this paper.

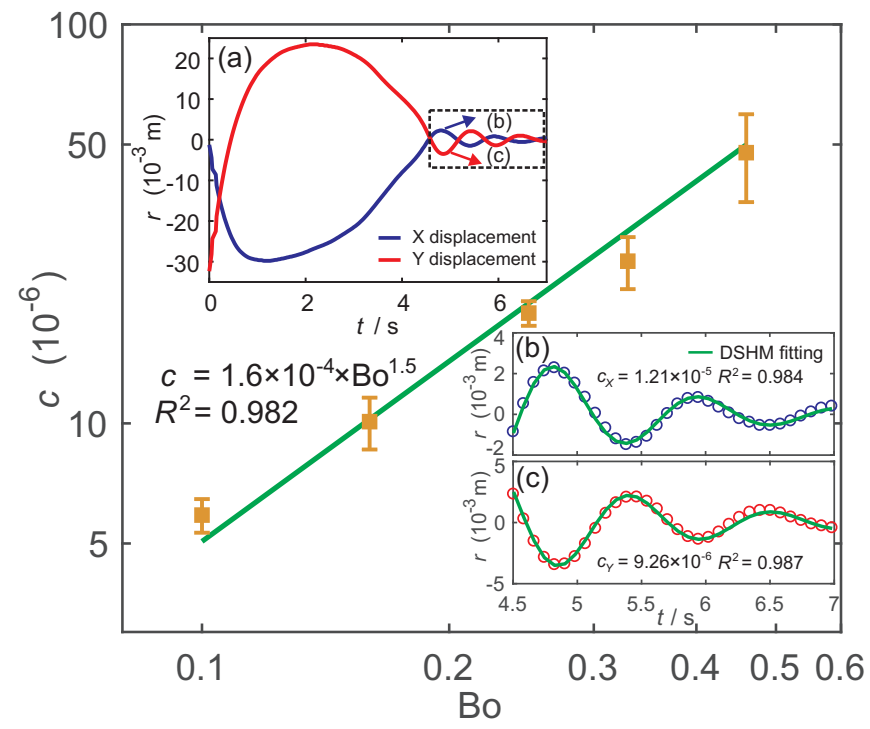

FIG. 4. The relationship of the viscous damping coefficient $c$ and the Bond number Bo of floating LMs in DEP trapping cases (logarithmic coordinates). Insets: (a) The trends of marble positions $r(x, y)$ versus time in a successful trapping case of a 5- $\mu$ L floating LM. (b),(c) The oscillation of the floating LM relative to the electrode center line and fitting with the solution of DSHM at the end of trapping process.

Assuming that the floating LM travels along an approximately straight path before reaching the critical position, which is confirmed by experimental marble trajectories (Figs. 3(a)-(d)), we can calculate the friction energy loss with dynamic data from the real-time detection of marble positions. This energy loss mainly represents the kinetic friction force $F_{k f}$ between the floating LM and the carrier liquid by Stokes friction with a correction factor $f(V, R, z)[36,37]$ and can be expressed as $U_{\text {loss }}=-\int_{i}^{c} F_{k f} d s=\int_{i}^{c} 6 \pi f \mu R v d s$. In a previous work [36], we have demonstrated that the term $6 \pi f \mu R$ of Stokes friction is analogous to the viscous damping coefficient $c$ of the well-known damped simple harmonic motion (DSHM) at the end of trapping process. The value of $c$ could be experimentally determined by analyzing the successful trapping cases of various LMs. We find that this coefficient is constant for each specific marble mass and is a function of the Bond number of the LM, Fig. 4. The initial velocity $v_{i}$ deduced from the average velocity of the first six video frames is used instead of the instantaneous velocity in the friction energy loss calculation (mostly within $0.25 \mathrm{~s}$ ). The initial marble position $r_{i}$ and injection angle $\theta$ are used to estimate the straight sliding distance. Based on the above equations, the general governing equation for DEP trapping of a floating LM is:

$$
\frac{1}{2} m v_{i c}^{2}+U_{\mathrm{DEP}, i}=c v_{i} r_{i} \cos \theta \text {. }
$$

Therefore, the magnitude of the initial critical velocity $v_{i c}$ could be further derived. This initial critical velocity can be used as the criterion to predict successful trapping. A floating LM sliding with an initial velocity close to $v_{i c}$ is more likely to escape from the DEP trap. In contrast, the marble would likely slip smoothly toward the position right below the electrode centroid in a spiral movement if the initial velocity is less than $v_{i c}$. In all trapping experiments with marble volumes ranging from 2.5 to $25 \mu \mathrm{L}$, we find that the analytical model described above can predict the experimental outcomes, no matter of trapping or escaping, with an accuracy of over $90 \%$ (Fig. 5). The good agreement indicates that the analytical model and the generalized governing equation are suitable for describing the DEP trapping of floating LMs. In Figs. 5(a)(c), all experimental outliers are close to the boundary line. Among these outliers, the successful trapping cases showing in the escaping region are attributed to the phenomenon that some floating LMs escape and then are trapped back if they stop within the effective working range of the DEP force.

\section{CONCLUSIONS AND PERSPECTIVES}

This trapping scheme via dielectrophoresis can be used for the manipulation of two or more floating LMs with a customized electrode array. A programable electrode array is key for the implementation of a LM-based digital microfluidic platform.

In conclusion, an effective DEP trapping method is proposed for moving and floating LMs with various volumes. Furthermore, we derive a governing equation for successful DEP trapping of LMs from the energy balance with parameters such as marble mass $m$, initial marble position $r_{i}$, initial 


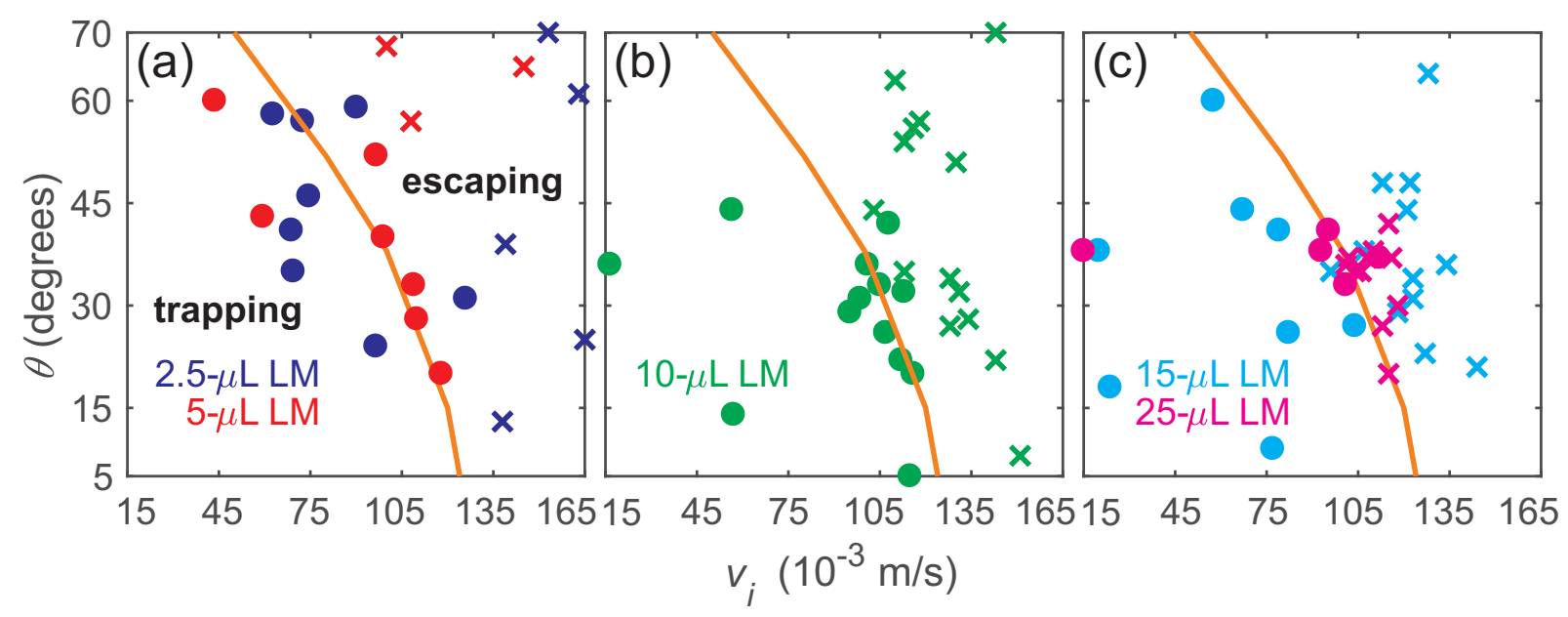

FIG. 5. Operation maps with an identical boundary (orange solid line) for DEP trapping of nonstationary floating LMs with various initial parameters. (a) Small marble volumes: 2.5 and $5 \mu \mathrm{L}$. (b) Medium marble volume: $10 \mu \mathrm{L}$. (c) Large marble volumes: 15 and $25 \mu \mathrm{L}$. Solid points represent the successful trapping in experiments, while solid crosses are escaping counterparts.

velocity $v_{i}$, injection angle $\theta$ and applied trapping voltage $V$. The result presented in this letter provides a practical engineering solution for LM transport and DEP manipulation of not only LMs but also other small soft matter.

\section{ACKNOWLEDGMENTS}

This work is performed in part at the Queensland Node of the Australian National Fabrication Facility, a company established under the National Collaborative Research Infrastructure Strategy to provide nano and micro-fabrication facilities for Australia's researchers. The authors acknowledge the Aus- tralian Research Council (ARC) for the grant of the Discovery Project (DP) DP170100277. J.J. and K.R.S. are supported by the Griffith University International Postgraduate Research Scholarship (GUIPRS) and the ARC DP grant, respectively.

\section{Appendix A: Trapping and escaping of floating liquid marbles under dielectrophoresis}

Videos 1 to 6 are the typical cases of floating liquid marbles trapped or escaping under the effect of DEP force (experimental parameters are listed in each caption). All videos are recorded at a rate of 125 frames/s and played at 60 frames/s.
[1] P. Aussillous and D. Quéré, Liquid marbles, Nature 411, 924 (2001).

[2] P. Aussillous and D. Quéré, Properties of liquid marbles, Proc. Royal Soc. A Math. Phys. Eng. Sci. 462, 973 (2006).

[3] E. Bormashenko, Liquid marbles: properties and applications, Curr. Opin. Colloid Interface Sci. 16, 266 (2011).

[4] E. Bormashenko, New insights into liquid marbles, Soft Matter 8, 11018 (2012).

[5] G. McHale and M. I. Newton, Liquid marbles: principles and applications, Soft Matter 7, 5473 (2011).

[6] J. Jin, C. H. Ooi, D. V. Dao, and N.-T. Nguyen, Coalescence processes of droplets and liquid marbles, Micromachines 8, 336 (2017).

[7] P. S. Bhosale, M. V. Panchagnula, and H. A. Stretz, Mechanically robust nanoparticle stabilized transparent liquid marbles, Appl. Phys. Lett. 93, 034109 (2008).

[8] M. Dandan and H. Y. Erbil, Evaporation rate of graphite liquid marbles: comparison with water droplets, Langmuir 25, 8362 (2009).

[9] A. Tosun and H. Erbil, Evaporation rate of ptfe liquid marbles, Appl. Surf. Sci. 256, 1278 (2009).

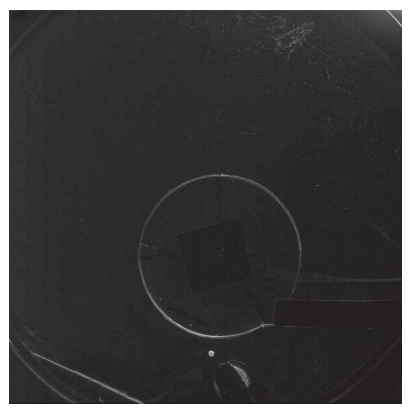

Video 1. A 2.5- $\mu \mathrm{L}$ LM trapped with an initial velocity of $69.5 \mathrm{~mm} / \mathrm{s}$ and an injection angle of $35^{\circ}$.

[10] Y. Zhao, J. Fang, H. Wang, X. Wang, and T. Lin, Magnetic liquid marbles: manipulation of liquid droplets using highly hydrophobic fe3o4 nanoparticles, Adv. Mater. 22, 707 (2010).

[11] H.-N. Polwaththe-Gallage, C. H. Ooi, J. Jin, E. Sauret, N.-T. Nguyen, Z. Li, and Y. Gu, The stress-strain relationship of liquid marbles under compression, Appl. Phys. Lett. 114, 043701 


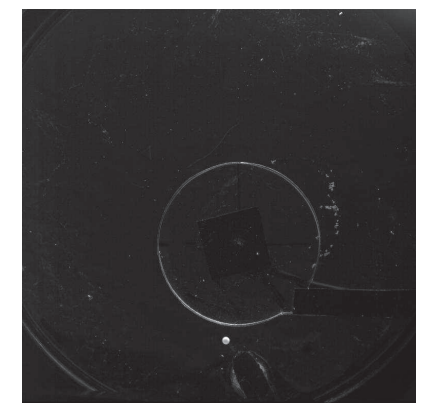

Video 2. A 5- $\mu \mathrm{L} \mathrm{LM}$ trapped with an initial velocity of $59.5 \mathrm{~mm} / \mathrm{s}$ and an injection angle of $43^{\circ}$.

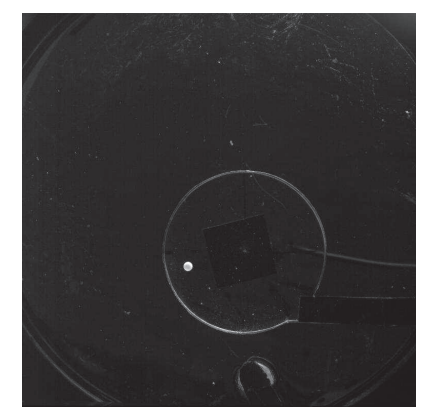

Video 3. A $10-\mu \mathrm{L}$ LM trapped with an initial velocity of $56.6 \mathrm{~mm} / \mathrm{s}$ and an injection angle of $45^{\circ}$.

(2019).

[12] H. Kawashima, M. Paven, H. Mayama, H.-J. Butt, Y. Nakamura, and S. Fujii, Transfer of materials from water to solid surfaces using liquid marbles, ACS Appl. Mater. Interfaces 9, 33351 (2017).

[13] J. Jin and N.-T. Nguyen, Manipulation schemes and applications of liquid marbles for micro total analysis systems, Microelectron. Eng. 197, 87 (2018).

[14] N.-T. Nguyen, M. Hejazian, C. H. Ooi, and N. Kashaninejad, Recent advances and future perspectives on microfluidic liquid handling, Micromachines 8, 186 (2017).

[15] E. Bormashenko and A. Musin, Revealing of water surface pollution with liquid marbles, Appl. Surf. Sci. 255, 6429 (2009).

[16] J. Tian, T. Arbatan, X. Li, and W. Shen, Liquid marble for gas sensing, Chem. Commun. 46, 4734 (2010).

[17] J. Tian, T. Arbatan, X. Li, and W. Shen, Porous liquid marble shell offers possibilities for gas detection and gas reactions, Chem. Eng. J. 165, 347 (2010).

[18] H. K. Lee, Y. H. Lee, I. Y. Phang, J. Wei, Y.-E. Miao, T. Liu, and X. Y. Ling, Plasmonic liquid marbles: A miniature substrateless sers platform for quantitative and multiplex ultratrace molecular detection, Angew. Chem. Int. Ed. 53, 5054 (2014).

[19] T. Arbatan, A. Al-Abboodi, F. Sarvi, P. P. Y. Chan, and W. Shen, Tumor inside a pearl drop, Adv. Healthc. Mater. 1, 467 (2012).

[20] J. Tian, N. Fu, X. D. Chen, and W. Shen, Respirable liquid marble for the cultivation of microorganisms, Colloids Surf. B Biointerfaces 106, 187 (2013).

[21] R. K. Vadivelu, C. H. Ooi, R.-Q. Yao, J. T. Velasquez, E. Pastrana, J. Diaz-Nido, F. Lim, J. A. Ekberg, N.-T. Nguyen, and J. A. St John, Generation of three-dimensional multiple spheroid model of olfactory ensheathing cells using floating liquid marbles, Sci. Rep. 5, 15083 (2015).

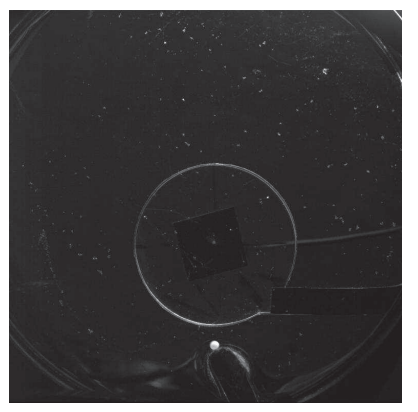

Video 4. A $10-\mu \mathrm{L}$ LM escapes with an initial velocity of $131 \mathrm{~mm} / \mathrm{s}$ and an injection angle of $33^{\circ}$.

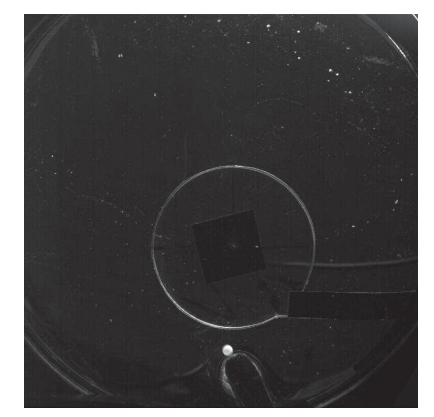

Video 5. A $15-\mu \mathrm{L}$ LM escapes with an initial velocity of $123 \mathrm{~mm} / \mathrm{s}$ and an injection angle of $34^{\circ}$.

[22] R. K. Vadivelu, H. Kamble, M. J. Shiddiky, and N.-T. Nguyen, Microfluidic technology for the generation of cell spheroids and their applications, Micromachines 8, 94 (2017).

[23] K. R. Sreejith, C. H. Ooi, J. Jin, D. V. Dao, and N.-T. Nguyen, Digital polymerase chain reaction technology-recent advances and future perspectives, Lab Chip 18, 3717 (2018).

[24] K. R. Sreejith, L. Gorgannezhad, J. Jin, C. H. Ooi, H. Stratton, D. V. Dao, and N.-T. Nguyen, Liquid marble as a biochemical reactor for polymerase chain reaction, Lab Chip 21, 28 (2019).

[25] G. McHale, D. Herbertson, S. Elliott, N. Shirtcliffe, and M. Newton, Electrowetting of nonwetting liquids and liquid marbles, Langmuir 23, 918 (2007).

[26] E. Bormashenko, Y. Bormashenko, A. Musin, and Z. Barkay, On the mechanism of floating and sliding of liquid marbles, ChemPhysChem 10, 654 (2009).

[27] L. Zhang, D. Cha, and P. Wang, Remotely controllable liquid marbles, Adv. Mater. 24, 4756 (2012).

[28] E. Bormashenko, R. Pogreb, R. Balter, O. Gendelman, and D. Aurbach, Composite non-stick droplets and their actuation with electric field, Appl. Phys. Lett. 100, 151601 (2012).

[29] S.-Y. Tang, V. Sivan, K. Khoshmanesh, A. P. O'Mullane, X. Tang, B. Gol, N. Eshtiaghi, F. Lieder, P. Petersen, A. Mitchell, et al., Electrochemically induced actuation of liquid metal marbles, Nanoscale 5, 5949 (2013).

[30] D. Zang, J. Li, Z. Chen, Z. Zhai, X. Geng, and B. P. Binks, Switchable opening and closing of a liquid marble via ultrasonic levitation, Langmuir 31, 11502 (2015).

[31] Y. Zhao, Z. Xu, H. Niu, X. Wang, and T. Lin, Magnetic liquid marbles: Toward "lab in a droplet", Adv. Func. Mater. 25, 437 (2015).

[32] M. Paven, H. Mayama, T. Sekido, H.-J. Butt, Y. Nakamura, and S. Fujii, Light-driven delivery and release of materials using 


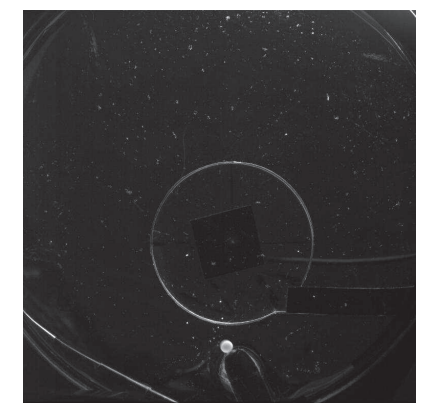

Video 6. A $25-\mu \mathrm{L}$ LM escapes with an initial velocity of $116 \mathrm{~mm} / \mathrm{s}$ and an injection angle of $37^{\circ}$.

liquid marbles, Adv. Func. Mater. 26, 3199 (2016).

[33] X. Fu, Y. Zhang, H. Yuan, B. P. Binks, and H. C. Shum, Controlled actuation of liquid marbles on a dielectric, ACS Appl. Mater. Interfaces 10, 34822 (2018).

[34] J. Jin, C. H. Ooi, D. V. Dao, and N.-T. Nguyen, Liquid marble coalescence via vertical collision, Soft matter 14, 4160 (2018).

[35] C. H. Ooi, J. Jin, A. V. Nguyen, G. M. Evans, and N.-T. Nguyen, Picking up and placing a liquid marble using dielectrophoresis, Microfluid. Nanofluidics 22, 142 (2018).

[36] J. Jin, C. H. Ooi, K. R. Sreejith, D. V. Dao, and N.-T. Nguyen, Dielectrophoretic trapping of a floating liquid marble, Phys. Rev. Appl. 11, 044059 (2019).

[37] C. H. Ooi, J. Jin, K. R. Sreejith, A. V. Nguyen, G. M. Evans, and N.-T. Nguyen, Manipulation of a floating liquid marble using dielectrophoresis, Lab Chip 18, 3770 (2018).

[38] J. Jin, C. H. Ooi, K. R. Sreejith, J. Zhang, A. V. Nguyen, G. M. Evans, D. V. Dao, and N.-T. Nguyen, Accurate dielectrophoretic positioning of a floating liquid marble with a twoelectrode configuration, Microfluid. Nanofluidics 23, 85 (2019).

[39] E. Bormashenko, R. Pogreb, G. Whyman, and A. Musin, Surface tension of liquid marbles, Colloids Surf. A Physicochem. Eng. Asp. 351, 78 (2009).

[40] E. Bormashenko, R. Pogreb, G. Whyman, A. Musin, Y. Bormashenko, and Z. Barkay, Shape, vibrations, and effective surface tension of water marbles, Langmuir 25, 1893 (2009).

[41] E. Bormashenko, A. Musin, G. Whyman, Z. Barkay, A. Starostin, V. Valtsifer, and V. Strelnikov, Revisiting the surface tension of liquid marbles: Measurement of the effective surface tension of liquid marbles with the pendant marble method, Colloids Surf. A Physicochem. Eng. Asp. 425, 15 (2013).

[42] C. H. Ooi, A. Van Nguyen, G. M. Evans, O. Gendelman, E. Bormashenko, and N.-T. Nguyen, A floating self-propelling liquid marble containing aqueous ethanol solutions, Rsc Adv. 5, 101006 (2015).

[43] C. H. Ooi, R. K. Vadivelu, J. St John, D. V. Dao, and N.-T. Nguyen, Deformation of a floating liquid marble, Soft matter 11, 4576 (2015).

[44] T. Arbatan and W. Shen, Measurement of the surface tension of liquid marbles, Langmuir 27, 12923 (2011). 